\title{
Eficiencia del sector bancario en Chile ante cambios en las regulaciones de protección al consumidor financiero*
}

\section{Efficiency of the banking sector in Chile in the face of changes in financial consumer protection regulations} Eficiência do sector bancário no Chile diante das mudanças nas relações de proteção os consumidor financeiro

Luis Cofré Sepúlveda

Universidad del Bio-Bio, Chile

ORCID: http://orcid.org/0000-0002-6175-2973

Luis Améstica-Rivas ${ }^{\text {a }}$

Universidad del Bio-Bío, Universidad Politécnica de

Cataluña, Chile

lamestica@ubiobio.cl

ORCID: http://orcid.org/0000-0003-0482-0287

Andrea King-Dominguez

Universidad del Bío-Bío, Chile

ORCID: http://orcid.org/0000-0002-1063-4336

Rodrigo Romero-Romero

Universidad del Bio-Bio, Chile

ORCID: http://orcid.org/0000-0001-5387-8616
DOI: https://doi.org/10.11144/Javeriana.cc20-50.esbc

Fecha de recepción: 14 Octubre 2018

Fecha de aprobación: 04 Octubre 2019

Fecha de publicación: 30 Noviembre 2019

\section{Resumen:}

En el presente estudio se analiza la eficiencia del sector bancario chileno entre los años 2010-2014. Este periodo contempla la promulgación de las nuevas regulaciones de protección al consumidor financiero del año 2012, que se supone generaron mayor competencia y simetrías de información en el mercado bancario. Metodológicamente se utilizó la técnica no paramétrica de análisis envolvente de datos, midiendo la eficiencia técnica global, la eficiencia técnica pura y la eficiencia a escala. Como resultado se obtuvo un promedio general de eficiencia del sector bancario chileno del 85,74\%, dejando espacio para un mejor desempeño, siendo el Banco Estado el de mejor eficiencia bajo estos parámetros. A su vez, a partir de los cambios reguladores en favor del consumidor financiero, se observó en 2012 un deterioro en la eficiencia y una recuperación un poco más lenta en los años posteriores, lo que en parte puede ser explicado por la entrada en vigencia de la ley.

Códigos JEL: G10, G21, G28.

Palabras clave: Bancos, eficiencia, análisis envolvente de datos, regulación financiera, Chile.

\section{Abstract:}

The current study analyzes the efficiency of the Chilean banking sector between 2010-2014. This period includes the issuance of new regulations for financial consumer protection in 2012, which are expected to have generated greater competition and information symmetries in the banking market. Methodologically, the non-parametric technique of data enveloping analysis was used, measuring global technical efficiency, pure technical efficiency and efficiency on scale. As a result, an overall average efficiency of the Chilean banking sector of $85.74 \%$ was obtained, leaving space for better performance, with Banco Estado being the most efficient under these parameters. In turn, from the regulatory changes in favor of the financial consumer, there was a deterioration in efficiency in 2012, a slightly slower recovery in subsequent years, which in part can be explained by the entry into force of the law.

JEL Codes: G10, G21, G28.

Keywords: Banks, efficiency, data envelopment analysis, financial regulation, Chile.

Notas de autor:

a Autor correspondencia. Correo electrónico: lamestica@ubiobio.cl 


\section{Resumo:}

Este estudo analisa a eficiência do setor bancário chileno entre 2010-2014. Esse período inclui a promulgação de novas regulamentações para a proteção do consumidor financeiro em 2012 que deveriam ter gerado maior concorrência e simetrias de informação no mercado bancário. Metodologicamente, foi utilizada a técnica não paramétrica de análise por envoltória de dados, medindo eficiência técnica global, eficiência técnica pura e eficiência à escala. Como resultado, obteve-se uma eficiência média geral do setor bancário chileno de 85,74\%, deixando espaço para um melhor desempenho, sendo o Banco Estado o mais eficiente sob esses parâmetros. Por sua vez, a partir das mudanças regulatórias em favor do consumidor financeiro, houve uma deterioração da eficiência em 2012, uma recuperação ligeiramente mais lenta nos anos seguintes, o que em parte pode ser explicado pela entrada em vigor da lei.

Códigos JEL: G10, G21, G28.

Palavras-chave: Bancos, eficiência, análise envoltória de dados, regulação financeira, Chile.

\section{INTRODUCCIÓN}

La solvencia bancaria es sumamente importante para la economía del país. En la literatura económica, diversas investigaciones demuestran el impacto directo que tiene el desarrollo de la actividad financiera en el crecimiento económico, por ejemplo Fitzgerald (2007), afirmó que "el desarrollo financiero y el crecimiento económico están claramente relacionados, y dicha relación ha estado presente en la mente de los economistas, desde Smith a Schumpeter"; por esto se espera que su funcionamiento sea eficiente y, además, esté alineado con los modelos económicos operantes en la actualidad.

El presente estudio se focaliza en la medición de la eficiencia, definida como el grado en el cual un sistema realiza sus funciones con un mínimo costo y/o consumo de recursos (Coll \& Blasco, 2006). La medición de la eficiencia se puede abordar a través de diferentes enfoques; el primero consiste en el estudio de las economías de escala y alcance, donde se estiman funciones de costos medios, y se supone implícitamente que la única fuente de ineficiencia es la asociada al tamaño y a la combinación de outputs. Bajo esta perspectiva, una forma de mejorar la eficiencia de una entidad consistiría en buscar la dimensión más adecuada, es decir, aquella que se asocie con el mínimo costo medio. Un segundo enfoque consiste en el análisis a través de indicadores parciales de productividad, donde se relaciona una medida del output producido con uno de los recursos utilizados en su consecución. Por otra parte, la mayoría de los trabajos que abordan el estudio de la eficiencia se inclinan por la estimación de fronteras eficientes y la evaluación de las ineficiencias-X. Un factor identificado en la literatura económica como determinante de las diferencias de eficiencia es la ineficiencia-X de Leibenstein (Server \& Melián, 1999).

Farrell (1957) propuso la primera descomposición de la eficiencia global en dos componentes diferenciados: la eficiencia técnica y la eficiencia asignativa, y que se justifican por la existencia de fallos de gestión o asignación incorrecta de recursos. Estas medidas de eficiencia han sido extendidas y aplicadas a unidades productivas por Charnes, Cooper, y Rhodes (1978). La principal técnica que estima la eficiencia en la metodología no paramétrica es el análisis envolvente (Data Envelopment Analysis, DEA).

El objetivo de este estudio es estimar la eficiencia económica global y determinar qué bancos son efi cientes y cuales están bajo el criterio de eficiencia en el sistema bancario chileno. En este sentido, la Superintendencia de Bancos e Instituciones Financieras de Chile-SBIF- realiza una medición de eficiencia bancaria mediante el ratio entre los gastos operacionales a ingresos operacionales resultando ser demasiado restringido como medida de eficiencia. Se utiliza un análisis mediante el enfoque no paramétrico DEA que permite una mejor medida de la eficiencia del sector bancario. El enfoque DEA es una aproximación para medir la ineficiencia usando técnicas de programación lineal (Charnes, Cooper, \& Rhodes, 1981), que no requiere una especificación particular de la frontera de producción efi ciente.

Dentro de las investigaciones que han aplicado el DEA a la banca se tiene la de Al-Faraj, Alidi, y BuBshait (1993), quienes estudiaron un conjunto de sucursales de uno de los mayores bancos comerciales de Arabia Saudita. Sathye (2001) comparó la eficiencia de los bancos australianos con la de la banca mundial en la década de 1990 y encontró que la eficiencia de los bancos australianos es inferior a la del promedio del 
resto del mindo. Otros estudios que abordan la eficiencia en el sector bancario son Athanassopoulos y Giokas (2000), Jemric y Vujcic (2002), Portela y Thanassoulis (2007), Chortareas, Girardone, y Ventouri (2013), los cuales realizaron análisis de eficiencia de diferentes bancos de Europa, mientras que Sathye (2001), Drake y Hall (2003), Ariff y Can (2008), Kong, Fu, y Yu (2016) y Chen y Liu (2017), aplicaron el DEA a bancos de China, Japón y Taiwán. Para América Latina se tienen los estudios de Asmild, Bogetoft, y Hougaard, (2013), Torres, Navarro y Gómez (2010), Gómez (2012), Canales (2013), Pirateque, Pireños, y Mondragón (2013), Gómez y Ríos (2016). Kong, Fu, y Yu (2016) evalúan la eficiencia de los bancos taiwaneses, tanto en la etapa operativa como en la de rentabilidad, en las actividades bancarias sobre la base de una muestra de 35 bancos nacionales en Taiwán en 2007. Los resultados indican que la eficiencia operacional fue menor que la eficiencia de rentabilidad.

En Chile, la Superintendencia de Bancos e Instituciones Financieras de Chile -SBIF- realiza una medición de eficiencia bancaria, dada por el ratio entre los gastos operacionales sobre los ingresos operacionales, pero es demasiado restringida como para poder tomarla como definitiva; por esto se han realizado investigaciones para determinar la eficiencia de la banca. Aguirre, Herrera y Bravo (2007) comparan la eficiencia técnica de la banca chilena y alemana, mediante fronteras estocásticas de producción y costo y DEA. En ambas fronteras estocásticas se utilizó una tecnología translogarítmica. Con el DEA se calculó el índice de Malmquist para analizar el cambio tecnológico (Malmquist, 1953). Los resultados indican que los bancos chilenos son altamente eficientes, tanto en costos (alrededor de 80\%) como en producción (bordea el 90\%). La banca alemana presenta un 90\% de eficiencia, tanto en costos como en producción y, resultó ser más eficiente que la banca chilena en un $15 \%$ en producción y $18 \%$ en costos. El índice de Malmquist muestra que el cambio tecnológico se ha mantenido más o menos constante en ambas bancas.

Por otra parte, Rodríguez y Rodríguez (2014) analizan la eficiencia en la generación de rentabilidad de los bancos comerciales que operan en México y Chile, así como su comportamiento en periodo de crisis. En particular, pretenden determinar si en periodo de crisis los bancos son más eficientes en la utilización de recursos para generar utilidades sobre los activos totales y sobre su patrimonio que en los periodos normales. Finalmente, se puede concluir que existe evidencia que los bancos de mayor eficiencia del periodo completo de análisis se concentran en el desempeño de los periodos de crisis, desde el punto de vista del resultado medido como el retorno sobre activos (ROA, por sus siglas en inglés) y el retorno sobre patrimonio (ROE, por sus siglas en inglés). En la mayoría de los casos, los bancos que alcanzaron mayor eficiencia en sus utilidades en el periodo previo a la crisis tienen un desempeño menos eficiente al compararlos con los mejores del tiempo de crisis. Los autores incluyen un conjunto limitado de variables relacionadas al cálculo de la rentabilidad como input y output en el estudio de la eficiencia, dejando de lado variables relativas a la organización tales como número de empleados y cantidad de sucursales.

El presente estudio utilizó el análisis DEA considerando la totalidad de los participantes en el sector bancario chileno, para a su vez verificar si existe un cambio en la eficiencia ante modificación de normas que influyen en el funcionamiento del mercado y así verificar qué participantes de este sector serán más competitivos y eficientes en el uso de sus recursos para poder entregar el mejor servicio con una mejor eficiencia. A diferencia de los estudios anteriores, se evalúa el desempeño a partir de cambios en la regulación del sector, incluyendo variables de input y output relativas al tamaño de la organización y la intermediación.

Como resultados del estudio se determina que la eficiencia del sistema bancario chileno aumentó en el periodo 2010-2014, alcanzando una eficiencia promedio de 83,4\% y 92,3\% al año 2014 en los modelos CRS y VRS respectivamente. A partir de la entrada en vigencia de la ley que protege al consumidor fi nanciero el año 2012, se observa una disminución de un 8,2\% de la eficiencia en 2013 que puede ser atribuida a este factor.

El artículo incluye la revisión de literatura referida a la regulación bancaria, posteriormente la metodología utilizada, un apartado que presenta los resultados y su análisis, para terminar con las conclusiones del estudio. 


\section{REGULACIÓN BANCARIA}

El estudio se enmarca dentro de la teoría de la regulación de los bancos de la década de 1970, que tiene las variantes normativa y positiva (Posner, 1974; Peltzman, 1976). El enfoque normativo tiene como objetivo justificar la intervención del gobierno señalando fallos del mercado en el sector bancario y el tipo de regulación más eficiente. El enfoque positivo tiene su origen en la escuela de Chicago (Stigler, 1971; Posner, 1974; Peltzman, 1976), y tiene por objetivo proporcionar explicaciones económicas de por qué ocurre la regulación y proporciona el análisis del impacto de la regulación. El enfoque positivo involucra la teoría del interés privado, que a la vez se conforman de las teorías de captura, la teoría económica de la regulación, la teoría de la burocracia y el enfoque de financiamiento público (Koumbarakis, 2018).

El estudio aborda el impacto de las regulaciones que buscan la protección del consumidor. La literatura económica sobre protección del consumidor examina la regulación bancaria en términos de fallos del mercado originados por asimetrías de información y externalidades. A este respecto, Goodhart et al. (1988), sugieren que la protección del consumidor surge por dos razones: (1) por la existencia de fallos del mercado (teoría de fallos del mercado), o (2) debido a la selección adversa de un banco por sus clientes o la incapacidad de los consumidores para monitorear el banco, es decir, la existencia de información asimétrica.

Desde una perspectiva teórica, las predicciones sobre los efectos de la regulación y supervisión en los bancos no son claras. Hay dos puntos de vista generales que proporcionan predicciones contradictorias (Barth, Caprio, \& Levine, 2006). Desde la visión del interés público se sostiene que el gobierno actúa en interés del público y regula los bancos para promover la eficiencia en la industria bancaria y mejorar los fallos del mercado. Por el contrario, el punto de vista del interés privado sostiene que la regulación se utiliza para promover los intereses de unos pocos. De acuerdo con la visión de interés público, una regulación bien estructurada puede mejorar la eficiencia al fomentar la competencia entre los bancos y alentar la gobernanza efectiva de los administradores bancarios. Por el contrario, desde el punto de vista del interés privado se esperaría que la regulación impida la eficiencia, ya que limitaría a los bancos en la oferta de sus servicios. Esto implica que la regulación bancaria no desempeñará un papel activo en la mejora de la eficiencia bancaria, sino que limitará a los bancos a canalizar recursos a grupos de interés especiales. Dados estos dos puntos de vista opuestos, y con predicciones conflictivas similares basadas en la teoría económica sobre el impacto de regulaciones específicas como requisitos de capital sobre el desempeño del banco, los estudios empíricos se vuelven aún más importantes para ayudar a informar las decisiones de política.

La eficiencia que pueden tener las diversas instituciones financieras puede verse afectada en cierta medida por cambios regulatorios, estas modificaciones pueden ser negativas como lo plantean Chortareas, Girardone, y Ventouri (2013), quienes sugiere "que una interferencia excesiva del gobierno en las actividades de las instituciones financieras puede afectar adversamente la operación eficiente de los bancos”. Además, hacen referencia a la libertad económica y financiera que, junto a una buena gobernabilidad, tienden a mostrar mejores niveles de eficiencia por el lado de los costos. Pero a su vez Pasiouras (2008), plantea que factores como las leyes nacionales que protegen la propiedad privada, los sistemas judiciales y los bajos niveles de corrupción y expropiación que fueron capturados en su estudio, mejoran la eficiencia en el sector bancario.

La falta de regulación en los mercados puede provocar crisis y así lo mencionan en su investigación Gómez y Ríos (2016), donde hacen referencia a que la crisis financiera internacional de 2008, dejo\# en evidencia los fallos estructurales en la función de regulación de los mercados financieros. Además, cita los estudios de Jackson, Campbell, Madrian y Tufano (2009), Claessens (2009) y Stiglitz (2009), quienes señalan que una de las causas de estos fallos se relaciona con el proceso desregulatorio de la década de 1980.

Para Berger y Humphrey (1997, p. 22) "el principal objetivo de la desregulación ha sido mejorar la eficiencia, pero los resultados han sido mixtos”. De acuerdo con Berg et al. (1992), luego de la desregularización los bancos obtienen mayor eficiencia y productividad, que es la misma conclusión de Zaim (1995) sobre las instituciones turcas. Además menciona que la eficiencia bancaria en los EEUU no sufrió 
cambios producto de la desregulación de principios de la década de 1980 (Bauer et al., 1993; Elyasiani \& Mehdian, 1995). Más recientemente, Gómez (2012) hace referencia a resultados obtenidos por Ahmed, Farooq, \& Jalil (2009) donde analizan las reformas implementadas en Pakista\#n y el impacto que causaron en el sistema bancario de ese país. En el estudio se incorpora el factor productivo a la metodología DEA. Es así como Alpay y Hassan (2007) realizaron una evaluación de la eficiencia del sistema bancario turco, luego de cambios estructurales en el sector financiero.

Según Contreras (2011), la protección de los consumidores tiene su origen en la adopción por parte del Congreso de los Estados Unidos, en 1968, de la Consumer Credit Protection Act (Ley de Protección al Consumidor Crediticio). Esta normativa asignaba a distintos órganos reguladores la aplicación de sus disposiciones, partiendo del tipo de entidad prestataria, entre ellas la Reserva Federal. En Europa, las primeras iniciativas en la materia tienen que ver con la autorregulación. En 1986 un grupo de bancos británicos estableció\# un "ombudsman" privado tras considerar las ventajas de reconocer las reglas protección a la clientela; más confianza, mayor y mejor información y la corrección de las desigualdades del mercado. Contreras (2011) menciona que la medida se adoptó\# luego de la publicación en 1983 del informe Banking Services and the Consumer del Consejo Nacional del Consumo británico. Esta tendencia fue seguida por países como España y Alemania y hoy en día es una práctica generalizada de los países de la Unión Europea.

Mendoza (2009) hace una comparación con América Latina y plantea que en Europa se comenzó a legislar y a favorecer a los clientes del sistema financiero a partir de la década de 1980 (Inglaterra 1985 y España 1986), en contraposición con la legislación para América Latina que comienza en la década de 1990, por ejemplo, Colombia en 1995 y México en 1999. Centroamérica, Panamá y El Salvador son países que cuentan con una legislación donde se explícita la protección a los usuarios del sistema financiero.

En Chile, en el año 2012 el enfoque regulatorio tomó una cierta tendencia a favorecer a los clientes-deudores, dándole mayor peso a este grupo, a expensas de los intereses de los depositantes. La Ley $\mathrm{n}^{\circ}$ 20555, la cual crea la normativa del Servicio Nacional del Consumidor Financiero (denominado Sernac Financiero), nace de la premisa de incorporar una mayor protección a los consumidores financieros, para los cuales existían factores que no podían manejar por la existencia de asimetrías. Estos factores son mejorar la información a la que pueden acceder los consumidores para la toma de sus decisiones de consumo en asuntos financieros, la creación de una unidad financiera especializada dentro del mismo servicio nacional del consumidor (SERNAC), donde se otorgar a un ministro de fe las facultades necesarias para certificar los hechos relativos al cumplimiento de la normativa contenida en la ley de protección al consumidor y, por otra parte la llamada Ley "no más Dicom", que regula el uso de los datos en los registros de los deudores del sistemafinanciero y comercial.

Por lo anterior, resulta interesante plantear la hipótesis de si la entrada en vigencia de la nueva legislación de protección al consumidor el año 2012, Ley del Sernac Financiero y Ley no más “Dicom”, influyeron en la eficiencia del sector bancario chileno y qué bancos fueron más eficientes a partir de su entrada en vigor.

\section{METODOLOGÍA}

En la medición de la eficiencia de una empresa es posible utilizar ratios financieros para medir el uso de los recursos. Otra posibilidad es utilizar el método de la frontera eficiente. Las alternativas disponibles para la estimación de la frontera eficiente son las técnicas paramétricas y las no paramétricas, denominadas también como estadísticas y matemáticas o de programación lineal, respectivamente. La frontera será paramétrica cuando se precise la definición previa de una determinada función, y no paramétrica cuando ésta no se requiera. Por otra parte, será determinística si supone que la distancia al nivel óptimo se debe exclusivamente a ineficiencias; son fronteras fijas en el espacio y sólo permiten valores ineficientes a un lado de la frontera. La principal técnica que estima la eficiencia en la metodología no paramétrica es el análisis envolvente de 
datos -DEA-, aunque también existe el Free Disposable Hull Analysis (FDH), pero es considerado como una variante del anterior. En cuanto a la metodología paramétrica hay tres aproximaciones a la frontera: la más utilizada, la estocástica (SFA, por sus siglas en inglés); la aproximación de libre distribución (DFA, por sus siglas en inglés), y la aproximación Thick Frontier Approach (Berger \& Humphrey, 1997; Server \& Melián, 1999; Kumbhakar, S., \& K. Lovell, 2000). En el caso de la metodología de frontera estocástica se requiere asumir una función específica a priori y la frontera se estima por mínimos cuadrados o máximum likelihood (Coelli, Rao, O’Donell, \& Battese, 2005).

Para determinar qué institución bancaria utilizó de mejor manera sus recursos disponibles, se utilizó el modelo DEA, debido a que, al ser un modelo no paramétrico, no necesita la especificación de una forma funcional, la cual traería complicaciones para determinar el sujeto de estudio. Lo importante del modelo es que realiza una comparación de distintas unidades solo con aquellas que poseen la misma o menor cantidad de insumos o recursos, lo que facilita la superación del problema de la diferencia en tamaño e ingresos de los bancos.

La metodología DEA es un modelo no paramétrico, que utiliza una técnica de programación lineal y permite comparar una relación entre entrada y salida de una o varias unidades productivas o de una empresa, estas unidades productivas (en adelante DMUs), que deben ser homogéneas, es decir, que utilicen los mismos inputs o insumos para producir la misma clase de outputs o resultados. La puntuación de eficiencia se expresa en valores que varían entre 0 y 1 , lo que permite también expresarlo en valores porcentuales.

Mediante el uso de este modelo se puede obtener la información de las distintas DMUs evaluadas y poder revisar si son o no eficientes. Si no son eficientes, indica en qué medida se deben disminuir los inputs o aumentar los outputs o modificar ambos, para alcanzar la eficiencia. En consecuencia, este análisis permite conocer a que DMUs eficientes deberían acercarse los DMUs no eficientes en términos de consumo de inputs y producción de outputs.

Con los resultados es posible construir una frontera eficiente, donde aquellas unidades productivas o DMUs que alcanzan el nivel máximo de outputs con los inputs utilizados se encuentran en la frontera, de manera que se puede determinar las DMUs que son ineficientes observando la distancia que se encuentran de la frontera.

De acuerdo con Charnes et al. (1981), el modelo se puede aplicar a través de dos enfoques:

1. Input orientados: dado un nivel de outputs, la máxima reducción proporcional en el vector de inputs mientras permanece en la frontera de posibilidades de producción. Una unidad no es eficiente si es posible disminuir cualquier input sin alterar sus outputs.

2. Output orientados: con el nivel de inputs, el máximo incremento proporcional de los outputs permaneciendo dentro de la frontera de posibilidades de producción. Se hace referencia a que una unidad no puede ser tratada como eficiente si es posible aumentar cualquier output sin incrementar ningún input y sin disminuir ningún otro output.

Con las orientaciones planteadas, se puede definir que una unidad será\# considerada eficiente si, y solo si, no es posible incrementar las cantidades de output manteniendo constantes las cantidades de inputs utilizadas, o bien es posible disminuir las cantidades de inputs empleadas sin modificar las cantidades de outputs obtenidas (Charnes et al., 1981). En la metodología DEA conviven variados modelos, de los cuales dos serán objeto de nuestro análisis. Las dos versiones más utilizadas son el modelo DEA-CCR que utiliza rendimientos constantes de escala, y el modelo DEA-BCC, el cual utiliza rendimientos variables de escala.

Para el modelo CCR, propuesto por Charnes, Cooper, y Rhodes (1978), el cual fue desarrollado a partir del trabajo de Farrell (1957), también conocido como CRS (Constant Returns to Scale), realiza el análisis considerando retornos constantes de escala, permitiendo determinar la eficiencia técnica global (ETG) de las DMUs. Tal como plantea Camelo, Coehlo y Borges (2011):

el modelo CCR considera ganancias de escala en el cálculo de la eficiencia, por lo tanto, la eficiencia relativa de una DMU es obtenida como el promedio de la división entre su productividad y la mayor productividad 
entre las DMUs analizadas en la observación. Con esto, el formato de la frontera de eficiencia del modelo CCR es una recta con un ángulo de 45 grados. (Camelo, Coehlo, \& Borges, 2011)

El modelo DEA en su variante CCR se expresa de la siguiente forma:

$$
\max _{(u, v)} h_{0}=\frac{\sum_{r=1}^{s} u_{r} y_{r 0}}{\sum_{i=1}^{m} v_{i} x_{i 0}}
$$

Sujeto a las siguientes restricciones:

$$
\begin{aligned}
& \frac{\sum_{r=1}^{s} u_{r} y_{r 0}}{\sum_{i=1}^{m} v_{i} x_{i 0}} \leq 1 \quad ; \quad j=1 \ldots n \\
& u_{r} v_{j} \geq 0 ; \quad r=1 \ldots \ldots s ; \quad i=1 \ldots \ldots m
\end{aligned}
$$

Donde en el numerador del cociente (1) se encuentra la suma de, que representa la cantidad de outputs $(1,2$, ..., $\mathrm{r}$ ) producidos por la unidad evaluada, multiplicados por los coeficientes de ponderación $\left(\mathrm{u}_{1}, \mathrm{u}_{2}, \ldots, \mathrm{u}_{\mathrm{r}}\right)$ que pueden ser considerados como un "precio" asociado al output $\left(\mathrm{y}_{10}, \mathrm{y}_{20}, \ldots .,\right)$. Por otro lado, en el denominador se encuentra la suma de xi0, que representa la cantidad de inputs $(1,2, \ldots, i)$ utilizados por la unidad evaluada en la producción de los outputs recogidos en el numerador, multiplicados por un coeficiente de ponderación $\left(\mathrm{v}_{1}, \mathrm{v}_{2}, \ldots, \mathrm{v}_{\mathrm{i}}\right)$ asignado por el programa, que representa el "precio" asociado a cada input correspondiente (x10, $\mathrm{x}_{20}, \ldots$, , ) y que es distinto para cada unidad. Así, cada vez que se estudia la eficiencia de una DMU, el programa tratará de encontrar el conjunto de "precios" $\left(\mathrm{u}_{\mathrm{r}}, \mathrm{v}_{\mathrm{i}}\right)$ que maximicen el valor del output de la unidad analizada con respecto al coste de sus inputs consumidos, resultando el ratio de eficiencia de cada DMU.

A partir de las ponderaciones $\left(\mathrm{u}_{\mathrm{r}}, \mathrm{v}_{\mathrm{i}}\right)$ para cada unidad de producción, las restricciones mencionadas pretenden asegurar que el cociente resultante de la ecuación (1) no sea superior a la unidad (1) para ninguna de las DMUs estudiadas. De esta forma, una DMU se considera eficiente cuando el resto de unidades no presentan una valoración superior a ella, alcanzando $\mathrm{h}_{0}$ el valor 1 ; siendo ineficientes aquellas otras DMUs que toman valores de h0 entre 0 y 1 .

El Modelo BCC denominado así por sus autores, (Banker, Charnes, \& Cooper, 1984), también conocido como VRS (Variable Returns to Scale), evalúa la eficiencia considerando retornos variables de escala, e indica el valor de la eficiencia técnica pura --ETP-. El modelo BCC, incorpora al modelo CCR los conceptos de economía de escala. Además, propone comparar DMUs que operan en escala similar. Así, la eficiencia de una DMU es obtenida dividiendo su productividad por la mayor productividad entre las DMUs con tamaño comparable. Este modelo no supone proporcionalidad entre entradas y salidas (Camelo et al., 2011).

Matemáticamente, para analizar la eficiencia utilizando el modelo BCC orientado ya sea, a los inputs o a los outputs se debe añadir la siguiente restricción a la fórmula del modelo CCR:

$$
\sum_{j=1}^{n} \lambda_{j}=1 ; \quad j=1, \ldots \ldots n
$$


Luego de haber obtenido tanto la eficiencia técnica global (ETG) como la eficiencia técnica pura (ETP), se puede determinar la eficiencia de escala $(\mathrm{EE})$, mediante la siguiente fórmula:

$$
E E=\frac{E T G}{E T P}
$$

Dado esto, se considera que la ineficiencia técnica pura surge del uso excesivo de recursos para la producción de un determinado nivel de outputs. Por otro lado, la ineficiencia de escala surge cuando la eficiencia técnica global es menor a la eficiencia técnica pura (Fernández \& Flórez, 2006). Para la resolución del modelo DEA se utilizó el software R (versión 3.5.1), mediante el paquete "Productivity".

\section{Variables y datos}

Para la identificación de las variables se debe plantear como lo menciona Avkiran (1999), Aguirre, Herrera y Bravo (2004), Chen y Liu (2017), que básicamente existen dos enfoques en la bibliografía sobre la definición del producto bancario: el enfoque de la intermediación y el enfoque de la producción (Humphrey, 1985). En el enfoque de la intermediación, el banco produce simultáneamente préstamos y depósitos, reconociendo en este último ciertas características que lo convierten en un producto específico. En este enfoque los insumos son sólo trabajo y capital.

En el enfoque de la producción, la firma utiliza capital y trabajo para producir distintas categorías de depósitos y préstamos. El producto se mide como el número de cuentas o como el número de transacciones por cuenta. Plantea como principal producto los servicios prestados a depositantes y como insumos: el trabajo y el capital físico. Sin embargo, varios autores, por falta de disponibilidad de información, utilizan los datos en unidades monetarias, bajo el supuesto que el aumento del valor de las operaciones implica un aumento de los costos operativos.

En la tabla 1 se resumen algunas de posturas, clasificadas por enfoque, además de las variables ocupadas por los estudios revisados. 
TABLA 1

Enfoques, métodos y variables de la literatura revisada

\begin{tabular}{|c|c|c|c|c|}
\hline Enfoque & Metoddogja & Autores & Variables Output & VariablesInput \\
\hline \multirow{13}{*}{ Istermediación } & \multirow{7}{*}{ DEA } & $\begin{array}{l}\text { W.H. Kong, T.-T.Fu \& M.-M. } \\
\text { Yu (2016) }\end{array}$ & $\begin{array}{l}\text { Préstamos. } \\
\text { Intersiones } \\
\text { Préstamos en mora } \\
\text { Ingresos por servicios. } \\
\text { Ingresos por intereses } \\
\text { Los ingresos por inversiones }\end{array}$ & $\begin{array}{l}\text { Gasto operacional, los foodos } \\
\text { prestables } \\
\text { stock de capital }\end{array}$ \\
\hline & & Ariff, Can L. 2008 & Prestamos totales e inversicnes & $\begin{array}{l}\text { Fondos prestables totales, tuumero } \\
\text { de empleados, capital fisicoy sus } \\
\text { precios }\end{array}$ \\
\hline & & dien Maomao, Liu Shiping (2017) & Préstamo, ganancia de libro & $\begin{array}{l}\text { Depósitos, gastos de operación, } \\
\text { cantidad de empleados }\end{array}$ \\
\hline & & Elyasiani y Meldian (1990) & \multirow{10}{*}{ Cartera de crédito e imversiones } & Depositos, $n^{\circ}$ de enpleados y capital \\
\hline & & Chansarn (2008) & & $\begin{array}{l}\text { Depósitos, cepital laboral y Costo } \\
\text { Total }\end{array}$ \\
\hline & & $\begin{array}{l}\text { Sarmiento, Cepeda, Mutis y Pere } \\
(2013)\end{array}$ & & $\begin{array}{l}\text { Depósitos, capital fisico, capital } \\
\text { Laboral y capital financiero }\end{array}$ \\
\hline & & Jemric y Vajeic (2002) & & \multirow[b]{2}{*}{$\begin{array}{l}\text { Depósitos, capital laboral y Activo } \\
\text { Fijo }\end{array}$} \\
\hline & \multirow{2}{*}{$\begin{array}{l}\text { Dea y froetera } \\
\text { estockisticas }\end{array}$} & $\begin{array}{l}\text { Fiorentino, Karman y Koetter } \\
(2006)\end{array}$ & & \\
\hline & & Zuñiga, S. Y Dap̧aino, E.(2003) & & $\begin{array}{l}\mathrm{N}^{*} \text { de emplados, capital financiero, } \\
\text { capital Fisico }\end{array}$ \\
\hline & DFA & Castro (2001) & & \multirow{3}{*}{$\begin{array}{l}\text { Depósito, capital de trabsjo y capital } \\
\text { fisico }\end{array}$} \\
\hline & \multirow{3}{*}{$\begin{array}{l}\text { Fronteras } \\
\text { estocísticas }\end{array}$} & Janna (2003) & & \\
\hline & & Cepeda, Srruiento y Mutis (2010) & & \\
\hline & & Estrada y Osonio (2004) & & $\begin{array}{l}\text { Depósitos, Capital Fisico y Capital } \\
\text { fnanciero }\end{array}$ \\
\hline \multirow{8}{*}{ Prodacción } & \multirow{8}{*}{ DEA } & $\mathrm{Yui}$ & \multirow{5}{*}{$\begin{array}{l}\text { Ingresos por intereses y otros ingresos } \\
\text { (operacionales } y \text { comisiones por } \\
\text { servicios) }\end{array}$} & Eqresos por intereses \\
\hline & & Jetinc y vajac (z00) & & Comisiones por servicios \\
\hline & & Chansam (2008) & & Gasto en Personal \\
\hline & & & & Gasto en capital \\
\hline & & Banker, Chang y Lee (2010) & & Otros eqresos operacionales \\
\hline & & Yang (2009) & $\begin{array}{l}\text { Nümero de Transacciones para gendar } \\
\text { diferentes trasacciones y procesos en } \\
\text { las sucursales del banco }\end{array}$ & Venta servicios, apoyo y otros \\
\hline & & $\begin{array}{l}\text { Kristianpoller Rodrigue: W, } \\
\text { Sawedra Rodriguez O (2014) }\end{array}$ & ROA Y ROE & $\begin{array}{l}\text { Gastos por inteeses del ejercicio, } \\
\text { Gastos de apoyo por el total de } \\
\text { activos }\end{array}$ \\
\hline & & $\begin{array}{l}\text { M. Seffino, D. Hoyos Maldcoado } \\
(2016)\end{array}$ & $\begin{array}{l}\text { Cactidades de cuentas corrientes, cajas } \\
\text { de alorro, placos fijos, préstam os y } \\
\text { tarjetas de crédito, indicador de ingresos } \\
\text { por servicios }\end{array}$ & $\begin{array}{l}\text { Cantidad de empleados, sucursales, } \\
\text { indicador de egresos por servicios }\end{array}$ \\
\hline
\end{tabular}

Fuente: elaborado a partir de Pirateque et al. (2013) y Chen y Liu, (2017).

Para el desarrollo de esta investigación se adoptó el enfoque de la intermediación, en la cual los bancos intermedian principalmente fondos entre ahorradores e inversores, y esto se adapta mejor a la evaluación del sujeto de análisis. Los datos utilizados para la investigación provienen de los estados financieros de los bancos comerciales en Chile incluidos en la base de datos de la Superintendencia de bancos e instituciones financieras (SBIF), organismo que supervisa todo el sistema financiero del país. La muestra del estudio comprende los 22 bancos comerciales (DMUs) que operaban en Chile durante el periodo 2010-2014. La muestra seleccionada se presenta en la tabla 2. 
TABLA 2

Bancos del estudio

\begin{tabular}{llll}
\hline \multicolumn{4}{c}{ Bancos operando en Chile } \\
\hline 1. Consorcio & 7. Penta & 13. HSBC Bank Chile & 19.Internacional \\
\hline 2. de Chile & 8. Ripley & 14. JP Morgan Chase Bank & 20.Corpbanca \\
\hline 3. Security & 9. de la Nación Argentina & 15. Rabobank Chile & 21. Itaú Chile \\
\hline 4. del Estado & 10. BBVA & 16. Santander Chile & 22. BICE \\
\hline 5. Do Brasil S.A. & 11. BCl & 17. Scotiabank Chile & \\
\hline 6. Falabella & 12. Deutsche Bank Chile & $\begin{array}{l}\text { 18. The Bank of Tokyo- } \\
\text { Mitsubishi }\end{array}$ & \\
\hline
\end{tabular}

Fuente: elaboración propia.

La selección de las variables se hizo con base en las investigaciones analizadas y el resumen de la tabla 1, y se determinaron tres variables inputs: $\mathrm{n}^{\circ}$ de empleados, $\mathrm{n}^{\circ}$ de sucursales y patrimonio. A su vez dos outputs fueron seleccionados: colocaciones y ROA para el cálculo de la eficiencia. En este sentido, el objetivo principal de esta investigación consiste en determinar que\# bancos se encuentran en la frontera de eficiencia, así\# como analizar las posiciones relativas del resto de DMUs y su evolución a lo largo del periodo analizado. Cabe mencionar que se posee como limitación en el análisis que existen variables que no se pueden controlar, además que cambios regulatorios pueden estar dentro de muchas medidas que ayudan o perjudican la eficiencia del sector.

\section{RESULTADOS}

Para saber la influencia que tienen los inputs en cada uno de los outputs, se realizó una matriz de correlación entre las variables. La tabla 3 muestra la relación entre las entradas y las salidas para los años de análisis.

TABLA 3

Coeficiente de correlación de variables, 2010-2014

\begin{tabular}{cccccc}
\hline Coeficientes & $\begin{array}{c}\text { Número } \\
\text { Sucursales }\end{array}$ & $\begin{array}{c}\text { Número } \\
\text { Empleados }\end{array}$ & Patrimonio & $\begin{array}{c}\text { Volumen } \\
\text { Colocaciones }\end{array}$ & ROA \\
\hline $\begin{array}{c}\text { Número } \\
\text { Sucursales }\end{array}$ & 1,000 & 0,967 & 0,799 & 0,928 & 0,384 \\
& $(0,000)^{*}$ & $(0,000)^{*}$ & $(0,000)^{*}$ & $(0,000)^{*}$ \\
\hline $\begin{array}{c}\text { Número } \\
\text { Empleados }\end{array}$ & 1,000 & 0,846 & 0,957 & 0,360 \\
\hline Patrimonio & & $(0,000)^{*}$ & $(0,000)^{*}$ & $(0,000)^{*}$ \\
\hline Volumen & & 1,000 & 0,853 & 0,367 \\
Colocaciones & & & $(0,000)^{*}$ & $(0,000)^{*}$ \\
\hline ROA & & 1,000 & 0,237 \\
& & & $(0,013)^{* *}$ \\
\hline
\end{tabular}


Se observa que la gran mayoría de las variables inputs tienen una alta correlación con respecto a las colocaciones, y una baja correlación con respecto a la Rentabilidad sobre los Activos. Destaca la variable de Colocaciones, la cual posee la más baja correlación con respecto a la Rentabilidad Sobre los Activos, donde alcanza un valor de un 0,237. Para este análisis y con base en los resultados podemos decir que existe una correlación positiva entre las variables.

\section{Eficiencia Técnica Global}

La Eficiencia Técnica Global es aquella que se obtiene al aplicar el modelo DEA bajo el supuesto de rendimientos constantes a escala. De los resultados obtenidos se observa que sólo seis entidades financieras de las 22 evaluadas, son las que lograron la eficiencia técnica global el año 2010 en comparación relativa con el conjunto de entidades que participan en este análisis. Si revisamos los años posteriores podemos observar que en el año 2011 se logra el máximo valor: diez unidades logran la eficiencia. En la tabla 4 se pueden observar los resultados completos obtenidos en el periodo analizado con la muestra total de los bancos.

TABLA 4

Resultados modelo CRS 2010-2014

\begin{tabular}{lrrrrr}
\hline \multicolumn{1}{c}{ BANCO } & $\mathbf{2 0 1 0}$ & $\mathbf{2 0 1 1}$ & $\mathbf{2 0 1 2}$ & $\mathbf{2 0 1 3}$ & \multicolumn{2}{c}{$\mathbf{2 0 1 4}$} \\
\hline Consorcio & 0,9880 & 0,4506 & 0,4456 & 0,7419 & 0,6016 \\
\hline de Chile & 0,8713 & 0,9056 & 0,8307 & 0,7954 & 0,7706 \\
\hline de la Nación Argentina & 0,0510 & 1 & 0,9000 & 0,0873 & 1 \\
\hline Estado & 0,9901 & 1 & 1 & 1 & 1 \\
\hline Do Brasil & 0,8675 & 1 & 1 & 0,5414 & 0,6014 \\
\hline Falabella & 0,6640 & 0,6995 & 0,7565 & 1 & 0,8100 \\
\hline Internacional & 1 & 1 & 0,9471 & 0,9999 & 0,9262 \\
\hline Itaú Chile & 0,6788 & 0,7817 & 0,7278 & 0,7946 & 0,8150 \\
\hline Penta & 0,3267 & 0,9434 & 0,9196 & 0,8216 & 1 \\
\hline Ripley & 1 & 1 & 1 & 0,6165 & 0,6665 \\
\hline Security & 1 & 1 & 1 & 1 & 0,9640 \\
\hline BBVA & 1 & 0,8045 & 0,8860 & 0,7493 & 0,7310 \\
\hline
\end{tabular}


TABLA 4_2

Resultados modelo CRS 2010-2014

\begin{tabular}{|c|c|c|c|c|c|}
\hline BANCO & 2010 & 2011 & 2012 & 2013 & 2014 \\
\hline $\mathrm{BCl}$ & 0,7974 & 0,8321 & 0,8067 & 0,7747 & 0,7682 \\
\hline $\mathrm{BICE}$ & 1 & 1 & 0,9564 & 1 & 1 \\
\hline Corpbanca & 0,9535 & 0,9110 & 0,9975 & 1 & 1 \\
\hline Deutsche Bank Chile & 0,6525 & 1 & 0,0623 & 1 & 0,9450 \\
\hline HSBC Bank Chile & 0,5178 & 1 & 1 & 0,6791 & 0,7495 \\
\hline JP Morgan Chase Bank & 1 & 1 & 1 & 0,3498 & 1 \\
\hline Rabobank Chile & 0,6355 & 0,6714 & 0,9636 & 1 & 1 \\
\hline Santander Chile & 0,8169 & 0,8308 & 0,8029 & 0,8174 & 0,8059 \\
\hline Scotiabank Chile & 0,6446 & 0,6719 & 0,7037 & 0,7058 & 0,7477 \\
\hline The Bank of Tokyo-Mitsubishi & 0,2903 & 0,2957 & 0,3729 & 0,5797 & 0,4658 \\
\hline Promedio & 0,7612 & 0,8544 & 0,8218 & 0,7752 & 0,8349 \\
\hline Mínimo & 0,0510 & 0,2957 & 0,0623 & 0,0873 & 0,4658 \\
\hline Máximo & 1 & 1 & 1 & 1 & 1 \\
\hline
\end{tabular}

Fuente: elaboración propia

En la tabla 5 se presenta el resumen de las instituciones que obtuvieron eficiencia técnica global, de la cual podemos deducir que solo el Banco Estado, BICE y JP Morgan Chase Bank logran en 4 de los 5 periodos mantener la ETG.

TABLA 5

Resumen Instituciones eficientes modelo CRS 2010-2014

\begin{tabular}{lllll}
\hline \multicolumn{1}{c}{2010} & \multicolumn{1}{c}{2011} & \multicolumn{2}{c}{2013} & 2014 \\
\hline Internacional & $\begin{array}{l}\text { de la Nación } \\
\text { Argentina }\end{array}$ & del Estado & del Estado & $\begin{array}{l}\text { de la Nación } \\
\text { Argentina }\end{array}$ \\
Ripley & del Estado & Do Brasil S.A. & Falabella & del Estado \\
Security & Do Brasil S.A. & Ripley & Security & Penta \\
BBVA & Internacional & Security & BICE & BICE \\
BICE & Ripley & HSBC Bank Chile & Corpbanca & Corpbanca \\
JP Morgan Chase & Security & JP Morgan Chase & Deutsche Bank & JP Morgan Ch \\
Bank & Bank & Chile & Bnak \\
& BICE & & Rabobank Chile & Rabobank Chi \\
& Deutsche Bank Chile & & & \\
& HSBC Bank Chile & & & \\
& JP Morgan Chase Bank & & & \\
\hline
\end{tabular}

Fuente: elaboración propia

El Banco Ripley mantiene la ETG en los años 2010 y 2012, pero en adelante presentó un déficit comparativo de 38,34\% en el año 2013 y 33,34\% en el año 2014. Otro caso que podemos observar es el de Deutsche Bank Chile el cual posee una ETG en los años 2011 y 2013 pero en los años 2010, 2012 y 2014 obtuvo como resultado un déficit comparativo de $34,74 \%, 93,76 \%$ y $5,5 \%$, respectivamente. En el caso de JP 
Morgan Chase Bank, obtiene una ETG de 100\% en casi todo el periodo de análisis, excepto en el año 2013 donde posee un déficit comparativo de un $65,02 \%$.

Si observamos la tabla 6 , que nos muestra todos aquellos bancos que poseen una ineficiencia técnica global, encontramos que el Banco de Tokio-Mitsubishi mantiene una ineficiencia menor al $50 \%$ en casi todos los periodos de análisis, aunque cabe mencionar que el año 2013 logra su mejor desempeño con un déficit comparativo $42,03 \%$.

TABLA 6

\begin{tabular}{|c|c|c|c|c|}
\hline 2010 & 2011 & 2011 & 2013 & 2014 \\
\hline Consorcio & Consorcio & Consorcio & Consorcio & Consorcio \\
\hline de Chile & de Chile & de Chile & de Chile & de Chile \\
\hline de la Nación & Falabella & de la Nación & de la Nación & Do Brasil S.A. \\
\hline del Estado & Itaú Chile & Falabella & Do Brasil S.A. & Falabella \\
\hline Do Brasil S.A. & Penta & Internacional & Internacional & Internacional \\
\hline Falabella & BBVA & Itaú Chile & Itaú Chile & Itaú Chile \\
\hline Itaú Chile & $\mathrm{BCl}$ & Penta & Penta & Ripley \\
\hline Penta & Corpbanca & BBVA & Ripley & Security \\
\hline $\mathrm{BCl}$ & Rabobank Chile & $\mathrm{BCl}$ & BBVA & BBVA \\
\hline Corpbanca & Santander Chile & BICE & $\mathrm{BCl}$ & $\mathrm{BCl}$ \\
\hline Deutsche Bank Chile & Scotiabank Chile & Corpbanca & HSBC Bank Chile & Deutsche Bank Chile \\
\hline HSBC Bank Chile & Tokyo-Mitsubishi & Deutsche Bank Chile & $\begin{array}{l}\text { JP Morgan Chase } \\
\text { Bank }\end{array}$ & HSBC Bank Chile \\
\hline Rabobank Chile & & Rabobank Chile & Santander Chile & Santander Chile \\
\hline Santander Chile & & Santander Chile & Scotiabank Chile & Scotiabank Chile \\
\hline Scotiabank Chile & & Scotiabank Chile & Tokyo-Mitsubishi & Tokyo-Mitsubishi \\
\hline Tokyo-Mitsubishi & & Tokyo-Mitsubishi & & \\
\hline
\end{tabular}

Fuente: elaboración propia.

\section{Eficiencia Técnica Pura}

La Eficiencia Técnica Pura es aquella que se obtiene al aplicar el modelo DEA bajo el supuesto de rendimientos variables a escala. Mediante este análisis se observa que para el año 2010 fueron 13 las instituciones que obtuvieron un $100 \%$ de eficiencia y por tanto conformaron la frontera de producción eficiente. En 2011, esta cifra aumentó a 15, pero los dos años sucesivos disminuyó: en 2012, 13 instituciones y en 2013, 12 instituciones. En el último año de análisis hubo una recuperación y fueron 13 las instituciones que lograron la eficiencia ETP. Los resultados de la medición de ETP se pueden observar en la tabla 7. 
TABLA 7

Resultados modelo VRS, 2010-2014

\begin{tabular}{|c|c|c|c|c|c|}
\hline BANCO & 2010 & 2011 & 2012 & 2013 & 2014 \\
\hline Consorcio & 1 & 0,6973 & 0,5239 & 0,9018 & 1 \\
\hline de Chile & 1 & 1 & 1 & 1 & 1 \\
\hline de la Nación Argentina & 1 & 1 & 1 & 1 & 1 \\
\hline Estado & 1 & 1 & 1 & 1 & 1 \\
\hline Do Brasil & 1 & 1 & 1 & 1 & 1 \\
\hline Falabella & 0,7706 & 0,7434 & 0,7775 & 1 & 0,9437 \\
\hline Internacional & 1 & 1 & 1 & 1 & 1 \\
\hline Itaú Chile & 0,7433 & 0,8293 & 0,7601 & 0,9266 & 0,8729 \\
\hline Penta & 0,9514 & 1 & 0,9652 & 0,8216 & 1 \\
\hline Ripley & 1 & 1 & 1 & 0,6329 & 1 \\
\hline Security & 1 & 1 & 1 & 1 & 0,9869 \\
\hline BBVA & 1 & 1 & 0,8880 & 0,7502 & 0,7318 \\
\hline $\mathrm{BCl}$ & 0,9059 & 0,9073 & 0,9263 & 0,9352 & 0,9092 \\
\hline BICE & 1 & 1 & 1 & 1 & 1 \\
\hline Corpbanca & 1 & 1 & 1 & 1 & 1 \\
\hline Deutsche Bank Chile & 0,7730 & 1 & 0,0623 & 1 & 0,9450 \\
\hline HSBC Bank Chile & 0,5813 & 1 & 1 & 0,7254 & 0,8072 \\
\hline JP Morgan Chase Bank & 1 & 1 & 1 & 0,3498 & 1 \\
\hline Rabobank Chile & 0,7256 & 0,7559 & 1 & 1 & 1 \\
\hline Santander Chile & 1 & 1 & 1 & 1 & 1 \\
\hline Scotiabank Chile & 0,7101 & 0,7160 & 0,7146 & 0,7719 & 0,7944 \\
\hline The Bank of Tokyo-Mitsubishi & 0,9531 & 0,4151 & 0,3858 & 0,7276 & 0,4658 \\
\hline Promedio & 0,9187 & 0,9120 & 0,8638 & 0,8883 & 0,9298 \\
\hline Mínimo & 0,5812 & 0,4151 & 0,0623 & 0,3498 & 0,4658 \\
\hline Máximo & 1 & 1 & 1 & 1 & 1 \\
\hline
\end{tabular}

Fuente: elaboración propia.

Según los datos obtenidos podemos establecer que para el año 2010 la eficiencia mínima la obtiene HSBC Bank Chile con un 58,12\%, en el año 2011 este mínimo lo obtiene The Bank of Tokio-Mitsubishi con un resultado del 41,51\% de eficiencia. En el año 2012 el Deutsche Bank Chile obtiene un 6,2\% de eficiencia, siendo éste el resultado más bajo de todos los años analizados. Luego en el año 2013 el mínimo lo obtiene JP Morgan Chase Bank con una eficiencia del 34,98\%. En el año 2014 nuevamente The Bank of TokioMitsubishi obtiene la menor eficiencia con un $46,6 \%$.

La tabla 8 expone un resumen de las instituciones que lograron esta eficiencia destacando entre ellas el Banco del Estado, Banco de Chile, Banco de la Nación Argentina, Banco do Brasil S. A., BICE, Corpbanca, 
Banco Internacional y Santander, quienes obtuvieron eficiencia técnica pura en la totalidad del periodo analizado.

TABLA 8

Resumen Instituciones eficientes modelo VRS 2010-2014

\begin{tabular}{|c|c|c|c|c|}
\hline 2010 & 2011 & 2012 & 2013 & 2014 \\
\hline Consorcio & de Chile & de Chile & de Chile & Consorcio \\
\hline de Chile & $\begin{array}{l}\text { de la Nación } \\
\text { Argentina }\end{array}$ & $\begin{array}{l}\text { de la Nación } \\
\text { Argentina }\end{array}$ & $\begin{array}{l}\text { de la Nación } \\
\text { Argentina }\end{array}$ & de Chile \\
\hline $\begin{array}{l}\text { de la Nación } \\
\text { Argentina }\end{array}$ & del Estado & del Estado & del Estado & $\begin{array}{l}\text { del Estad de la } \\
\text { Nación Argentina }\end{array}$ \\
\hline del Estado & Do Brasil S.A. & Do Brasil S.A. & Do Brasil S.A. & del Estado \\
\hline Do Brasil S.A. & Internacional & Internacional & Falabella & Do Brasil S.A. \\
\hline Internacional & Penta & Ripley & Internacional & Internacional \\
\hline Ripley & Ripley & Security & Security & Penta \\
\hline Security & Security & BICE & BICE & Ripley \\
\hline BBVA & BBVA & Corpbanca & Corpbanca & BICE \\
\hline $\mathrm{BICE}$ & BICE & HSBC Bank Chile & $\begin{array}{l}\text { Deutsche Bank } \\
\text { Chile }\end{array}$ & Corpbanca \\
\hline Corpbanca & Corpbanca & $\begin{array}{l}\text { JP Morgan Chase } \\
\text { Bank }\end{array}$ & Rabobank Chile & $\begin{array}{l}\text { JP Morgan Chase } \\
\text { Bank }\end{array}$ \\
\hline $\begin{array}{l}\text { JP Morgan Chase } \\
\text { Bank }\end{array}$ & $\begin{array}{l}\text { Deutsche Bank } \\
\text { Chile }\end{array}$ & Rabobank Chile & Santander Chile & Rabobank Chile \\
\hline Santander Chile & $\begin{array}{l}\text { HSBC Bank Chile } \\
\text { JP Morgan Chase } \\
\text { Bank } \\
\text { Santander Chile }\end{array}$ & Santander Chile & & Santander Chile \\
\hline
\end{tabular}

Fuente: elaboración propia.

\section{Eficiencia a Escala}

Se calculó la Eficiencia a Escala (EE), la cual se obtiene mediante el cociente entre la eficiencia técnica global obtenida del modelo CRS y la eficiencia técnica pura obtenida del modelo VRS.

De acuerdo con los resultados obtenidos, podemos inferir que de las 22 instituciones financieras analizadas en el estudio en el año 2010, sólo seis lograron la eficiencia a escala. La tendencia a lo largo del tiempo es ascendente en cuanto a las instituciones que logran ser eficientes llegando a nueve instituciones en el año 2014. Existe un aumento en la eficiencia durante el año 2012 alcanzando un promedio de 95,23\% de eficiencia del total de las instituciones, donde el valor mínimo de eficiencia fue de $80,29 \%$. Luego de la entrada en vigencia de la ley se observa una reducción de la eficiencia, comparada con respecto a los años 2011 y 2012 , alcanzando 
un promedio de $88 \%$ en el año 2013. En la tabla 9 se presentan el detalle de los resultados del análisis para el periodo 2010-2014.

TABLA 9

Resultados modelo eficiencia a escala (EE), 2010-2014

\begin{tabular}{|c|c|c|c|c|c|}
\hline BANCO & 2010 & 2011 & 2012 & 2013 & 2014 \\
\hline Consorcio & 0,9880 & 0,6461 & 0,8505 & 0,8227 & 0,6015 \\
\hline de Chile & 0,8713 & 0,9056 & 0,8307 & 0,7954 & 0,7706 \\
\hline de la Nación Argentina & 0,9055 & 1 & 0,9000 & 0,0873 & 1 \\
\hline Estado & 0,8307 & 1 & 1 & 1 & 1 \\
\hline Do Brasil & 0,7954 & 1 & 1 & 0,5414 & 0,6014 \\
\hline Falabella & 0,7706 & 0,9409 & 0,9729 & 1 & 0,8583 \\
\hline Internacional & 0,0510 & 1 & 0,9471 & 0,9999 & 0,9262 \\
\hline Itaú Chile & 1 & 0,9425 & 0,9575 & 0,8575 & 0,9336 \\
\hline Penta & 0,9000 & 0,9434 & 0,9527 & 1 & 1 \\
\hline Ripley & 0,0873 & 1 & 1 & 0,9740 & 0,6665 \\
\hline Security & 1 & 1 & 1 & 1 & 0,9640 \\
\hline BBVA & 0,9901 & 0,8045 & 0,9978 & 0,9988 & 0,9988 \\
\hline $\mathrm{BCl}$ & 1 & 0,9170 & 0,8709 & 0,8284 & 0,8448 \\
\hline BICE & 1 & 1 & 0,9564 & 1 & 1 \\
\hline Corpbanca & 1 & 0,9110 & 0,9975 & 1 & 1 \\
\hline Deutsche Bank Chile & 1 & 1 & 1 & 1 & 1 \\
\hline HSBC Bank Chile & 0,8675 & 1 & 1 & 0,9361 & 0,9285 \\
\hline JP Morgan Chase Bank & 1 & 1 & 1 & 1 & 1 \\
\hline Rabobank Chile & 1 & 0,8881 & 0,9636 & 1 & 1 \\
\hline Santander Chile & 0,5414 & 0,8308 & 0,8029 & 0,8174 & 0,8059 \\
\hline Scotiabank Chile & 0,6013 & 0,9384 & 0,9847 & 0,9143 & 0,9412 \\
\hline The Bank of Tokyo-Mitsubishi & 0,7643 & 0,7124 & 0,9666 & 0,7967 & 1 \\
\hline Promedio & 0,8165 & 0,9264 & 0,9523 & 0,8805 & 0,9024 \\
\hline Mínimo & 0,0510 & 0,6462 & 0,8029 & 0,0873 & 0,6013 \\
\hline Máximo & 1 & 1 & 1 & 1 & 1 \\
\hline
\end{tabular}




\section{Eficiencia promedio del sector bancario}

La tabla 10 presenta el promedio y desviación estándar de cada modelo en el periodo analizado, con el fin de hacer una caracterización general de la situación de Chile con respecto a la eficiencia de los bancos, y del comportamiento entre un año y otro.

El sector bancario chileno muestra una media elevada cuando hablamos de eficiencia, en el modelo de retornos constantes a escala -CRS- se observa un aumento año a año, pero en el periodo 2012 y 2013 vemos una disminución en la eficiencia (ver tabla 10), pero en general sobre el $75 \%$. Es más, para los periodos analizados obtuvieron un promedio de $80,95 \%$ de eficiencia.

Si nos centramos en el modelo de retornos variables a escala -VRS-, al ser sólo nueve entidades bancarias las que tuvieron ineficiencias en los resultados, con un valor promedio de 90,25\% para el total de periodos, cabe mencionar que la mayoría de los valores se encuentran cerca de la eficiencia.

TABLA 10

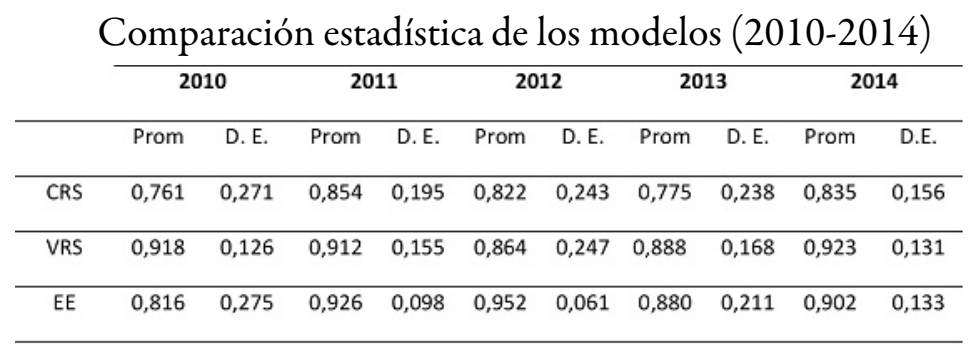

Fuente: elaboración propia.

Finalmente, los resultados de eficiencia a escala muestran valores sobre el $80 \%$ en todos los periodos, obteniendo como promedio una eficiencia que alcanza el 89,60\%, mientras que la desviación estándar es un poco menor del $15 \%$ gracias a la baja variación que existe en los resultados del modelo. Aunque son contextos y mediciones distintas, el resultado obtenido es coherente con el estudio de Candia, Aguirre, Orellana, y Gaete (2019), donde midieron los efectos de la crisis subprime en la banca chilena y donde los bancos tipificados como grandes llegaron a un nivel de eficiencia del 80,5\%.

\section{Análisis de los resultados}

Se observa que la eficiencia del sistema bancario chileno aumentó en el periodo 2010-2014, alcanzando una eficiencia promedio de $83,4 \%$ y $92,3 \%$ en el año 2014, según los modelos CRS y VRS respectivamente. La institución que poseen el mejor desempeño en eficiencia de acuerdo con ambos modelos es el Banco Estado con un promedio de 99,80\% y 100\%, en el modelo CRS y VRS, respectivamente. Cabe mencionar que esta institución es la tercera con mayor número de sucursales y números de empleados de los bancos analizados, superado por banco de Chile y Santander, respectivamente. El poseer una mayor cantidad de sucursales y empleados no asegura una eficiencia debido a que los bancos que siguen con mejor desempeño al Banco Estado son de menor tamaño. Estos bancos son BICE, Security y Corpbanca estimando sus niveles de eficiencia en promedio superiores al $98 \%$ en ambos modelos.

En relación con las instituciones bancarias que no lograron la eficiencia en ninguno de los tres modelos durante el periodo analizado destacan: Banco Itaú Chile, BCI, Scotiabank y The Bank of Tokio-Mitsubishi.

El Banco Itaú Chile obtiene resultados distintos con cada modelo. En el modelo CRS para el año 2014 obtiene el mejor desempeño alcanzando un nivel de eficiencia del 81,51\%; en el modelo VRS alcanza el mejor resultado en el año 2013 con 92,67\%; y con el modelo de eficiencia a escala el año 2012 obtiene la 
mejor combinación de input y output alcanzando un 95,75\%. Durante el periodo analizado, este banco se encontraba en expansión tanto en número de sucursales como en número de empleados. Aumentaron las colocaciones en un 42,22\%, pero el ROA sufrió una baja de un 20,96\% alcanzando una razón de 1,097\%. Esta condición de expansión puede explicar, en parte, la menor eficiencia de esta institución.

El banco BCI, según el modelo VRS, logró una eficiencia promedio de 91,68\%, alcanzando su máximo el año 2013 con un 93,52\%, pero disminuyendo el año siguiente a 90,92\%. En el modelo CRS obtiene una eficiencia promedio de un 79,58\%, donde la máxima eficiencia se logra el año 2011 con un 80,67\% y el mínimo el año 2014 con un 76,81\% y en comparación a la eficiencia a escala el cual su promedio fue de un $86,82 \%$. El banco BCI en el periodo analizado se encuentra en una serie de transformaciones, reduciendo el número de sucursales de 352 a 325 en 2014. Sin embargo, el número de empleados aumentó de 7012 en 2010 a 8427 empleados en 2014 (un aumento de 20,18\%), los montos de las colocaciones aumentaron en un 65,49\% y el ROA disminuyó de $1,67 \%$ a $1,44 \%$. El aumento de trabajadores y la disminución del número de sucursales tratando de mejorar su posicionamiento en el mercado, no logró mejorar su eficiencia. Es más, en el año 2014 tanto en modelo CRS y VRS obtuvo el menor desempeño en cuanto a eficiencia, siendo el año 2012 un punto de inflexión debido a que a partir de ese año comenzó la disminución de la eficiencia en los tres modelos. Aunque son eventos distintos, esta baja en la eficiencia coincide con otros shocks que han afectado a la banca chilena como ha sido la crisis subprime (Candia et al., 2019).

El banco de Tokio-Mitsubishi es el mayor banco de Japón, está presente en más de 40 países y en Chile sólo posee una sucursal en el periodo analizado. El número de empleados aumentó en 2014 al pasar de 42 a 64 , y el ROA obtenido aumentó de un $0,187 \%$ a un $0,55 \%$. De acuerdo con el modelo VRS la eficiencia fue disminuyendo en el tiempo desde $95,31 \%$ en 2010 hasta 46,58\% en 2014. En el modelo CRS el banco fue aumentando su eficiencia durante el periodo, comenzando con 29,03\% y alcanzando un 46,58\% en 2014. En resumen, el banco de Tokio-Mitsubishi obtuvo un 58,95\% (VRS), 40,09\% (CRS) y un 75,60\% (EE) en promedio para cada modelo, siendo la compañía con menor desempeño en el análisis.

La entrada en vigor de las leyes específicas que protegen al consumidor financiero en el año 2012 coincide con una reducción en la eficiencia a escala promedio durante el año 2013 para toda la muestra de bancos en un $8,2 \%$. Este efecto puede ser un factor adicional que influyó en la menor eficiencia de cada banco en particular. Este resultado atribuido a la mayor regulación, está en línea con los resultados obtenidos en otros estudios que encuentran resultados contradictorios, donde las restricciones a las actividades bancarias tienen impactos negativos sobre la eficiencia de costos, mientras que tienen efectos positivos en la eficiencia de ganancias (Pasiouras, Tanna, \& Zopounidis, 2009; Chortareas et al., 2013; Haque \& Brown, 2017).

\section{CONCLUSIONES}

En este artículo se analizó la eficiencia del sector bancario chileno aplicando los modelos de eficiencia técnica global -CRS- y eficiencia técnica pura -VRS-, que permiten establecer qué bancos presentaron mayor eficiencia en el periodo de estudio (2010-2014). En este sentido, se observa que la gran mayoría de las instituciones bancarias presentó una importante disminución en la eficiencia en el año 2012, que corresponde a la fecha de entrada en vigencia de la nueva legislación del sector financiero que protege al consumidor financiero ya sea, explicando y obligando a los bancos a transparentar la totalidad de la información al cliente, como también por la ley que regula el uso de datos en los registros de los deudores del sistema financiero y comercial en Chile.

Respecto a los resultados de eficiencia promedio del presente estudio de 80,95\% y 90,25\% según los modelo CRS y VRS respectivamente, no difieren significativamente con respecto a los obtenidos por Aguirre et al. (2007), quienes también plantearon que se requería una regulación que brindara a los bancos una mayor flexibilidad para que puedan aprovechar de mejor manera sus recursos. Además, los resultados del estudio 
incluyen un conjunto mayor de variables para la determinación de la eficiencia respecto a estudios anteriores en mercados financieros Latinoamericanos como Chile y México (Rodríguez \& Rodríguez, 2014).

No se puede afirmar que la regulación introducida en año 2012 sea la causa de la caída en la eficiencia del sector, pero se menciona como un hecho relevante en la memoria anual del SBIF la introducción de estas normativas que vienen a modificar el panorama bancario. Se determinó una disminución en la eficiencia en los años 2012 y 2013 en el sector bancario, que luego en año 2014 vuelve a subir. Entonces, no se podría asegurar que estas leyes sean la única causa de la reducción de la eficiencia en ese año, sin embargo, el sector bancario menciona como un hecho relevante -y que cambia las reglas del juego- la entrada en vigor de estas dos normativas.

Esta limitación puede dar paso a otros estudios que profundicen en identificar las variables que explican las ineficiencias dentro del sector. Tal como se señaló, estas regulaciones posiblemente afectaron al sistema bancario, pero en este escenario se hace evidente que los consumidores financieros, especialmente los clientes con menor acceso a información requieren protección y contar con información que minimice las asimetrías cuando acceden a productos financieros, situación extrapolable a otras realidades.

\section{Referencias}

Aguirre, M., Herrera, R., \& Bravo, G. (2004). Una frontera de producción para la banca chilena. Panorama socioeconómico, 29, 1-8.

Aguirre, M., Herrera, R., \& Bravo, G. (2007). Análisis comparativo de eficiencia técnica entre la banca chilena y alemana. Revista de Matemáticas: Teoría y Aplicaciones, 14 (2), 203-219.

Ahmed, U., Farooq, S., \& Jalil, H. (2009). Efficiency dynamics and financial reforms: Case study of Pakistani banks. International Research Journal of Finance and Economics, 25, 172-182. https://mpra.ub.unimuenchen.de/15054/

Al-Faraj, T. N., Alidi, A. S., \& Bu-Bshait, K. A. (1993). Evaluation of bank branches by means of data envelopment analysis. International Journal of Operations \& Production Management, 13(9), 45-52. https:// doi.org/10.1108/01443579310043628

Athanassopoulos, A., \& Giokas, D. (2000). The use of data envelopment analysis in banking institutions: Evidence from the commercial bank of Greece. Interfaces, 30(2), 81-95.

Ariff, M., \& Can, L. (2008). Cost and profit efficiency of Chinese banks: A non-parametric analysis. China Economic Review, 19(2), 260-273. https://doi.org/China Economic Review

Asmild, M., Bogetoft, P., \& Hougaard, J. (2013). Rationalising inefficiency: A study of Canadian Bank Branches. Omega, 41, 80-87.

Avkiran, N. K. (1999). An application reference for data envelopment analysis in branch banking: helping the novice researcher. International Journal of Bank Marketing, 17(5), 206-220. https:// doi.org/10.1108/02652329910292675

Banker, R., Charnes, A., \& Cooper, W. (1984). Some models for estimating technical and scale inefficiencies in data envelopment analysis management science. Management Science, 30(9), 1031-1142. https://doi.org/10.1287/ mnsc.30.9.1078

Barth, J., Caprio, G., Jr., \& Levine, R. (2006). Rethinking bank regulation: Till angels govern. Cambridge: Cambridge University Press.

Berger, A., \& Humphrey, D. (1997). Efficiency of financial institutions: International survey and directions for future research. European Journal of Operational Research, 98(2), 175-212. https://doi.org/10.1016/ S0377-2217(96)00342-6

Camelo, G., Coehlo, A., \& Borges, R. (2011). Eficiencia y benchmarking de operadores de ventas mediante el análisis envolvente de datos (DEA). Revista Electrônica Sistemas \& Gestâo, 6(1), 1-19. 
Canales, R. (2013). Analisis envolvente de datos\#: estimacion de la eficiencia técnica y asignativa del sector bancario nicaragüense. Revista Electrónica de Investigación en Ciencias Económicas, 1(1), 63-94.

Candia, C., Aguirre, M., Orellana, V., \& Gaete, M. (2019). La crisis subprime y sus efectos en la eficiencia del sector bancario chileno. Revista de Economia Institucional, 21(41), 187-211.

Charnes, A., Cooper, W., \& Rhodes, E. (1978). Measuring the efficiency of decision making units. European Journal of Operational Research, 2(6), 429-444. https://doi.org/10.1016/0377-2217(78)90138-8

Charnes, A., Cooper, W., \& Rhodes, E. (1981). Evaluating program and managerial efficiency: An application of data envelopment analysis to program follow through. Management Science, 27(6), 668-697. https:// doi.org/10.1287/mnsc.27.6.668

Chen, M., \& Liu, S. (2017). Analysis on the efficiency of Chinese listed commercial banks based on DEA. In Service Systems and Service Management (ICSSSM), 2017 International Conference on (pp. 1-5). IEEE.

Chortareas, G., Girardone, C., \& Ventouri, A. (2013). Financial freedom and bank efficiency: Evidence from the European Union. Journal of Banking and Finance, 37(4), 1223-1231. https://doi.org/10.1016/ j.jbankfin.2012.11.015

Coelli T., Rao D., O’Donell Ch.J., Battese, G. (2005). An introduction to efficiency and productivity analysis, 2nd ed. In C. Springer \& O. Blasco (2006). Evaluación de la eficiencia mediante el analisis envolvente de datos. Introducción a los modelos básicos. Universidad de Valencia.

Contreras, O. (2011). Consumidor bancario: fundamentos para un régimen especializado de protección. Documento de Trabajo, Federación Latianoamericana de Bancos FELABAN, República Dominicana.

Drake, L., \& Hall, M. (2003). Efficiency in Japanese banking: An empirical analysis. Journal of Banking and Finance, 27(5), 891-917. https://doi.org/10.1016/S0378-4266(02)00240-6

Farrell, M. J. (1957). The measurement of productive efficiency. Journal of the Royal Statistical Society. Series A (General). https://doi.org/10.2307/2343100

Fernández, Y., \& Flórez, R. (2006). Aplicación del modelo dea en la gestión pública. Un análisis de la eficiencia de las capitales de provincia españolas. Revista Iberoamericana de Contabilidad de Gestión, 7, 165-202.

Fitzgerald, V. (2007). Desarrollo financiero y crecimiento económico: una visión crítica. Principios, 1(7), 17-41.

Gómez, R. (2012). El sector bancario en México, los depósitos a plazo y las cuentas de ahorro: un análisis durante el periodo de internacionalización a través de la envolvente de datos ( DEA). Revista Ciencias Estratégicas, 20(27), 13-38.

Gómez, J., \& Ríos, O. (2016). Impacto de la gestión de riesgos sobre la eficiencia bancaria en costos, un caso aplicado a los bancos en Colombia. Cuadernos de Administración (Universidad del Valle), 32(56), 36-49.

Goodhart, C.A. (1988). The Evolution of Central Banks. Cambridge, Massachusetts and London: The MIT Press.

Haque, F., \& Brown, K. (2017). Bank ownership, regulation and efficiency: Perspectives from the Middle East and north Africa (MENA) region. International Review of Economics \& Finance, 47, 273-293.

Jemric, I., \& Vujcic, B. (2002). Efficiency of Banks in Croatia: A DEA Approach. Comparative Economic Studies, 44(2-3), 169-193. https://doi.org/10.1057/ces.2002.13

Kong, W.-H., Fu, T.-T., \& Yu, M.-M. (2016). Evaluating Taiwanese bank efficiency using the two-stage range DEA Model. International Journal of Information Technology \& Decision Making, 16, 1-26. https://doi.org/10.1142/ S0219622017500031

Koumbarakis, A. (2018). The economic theory of bank regulation and the redesign of Switzerland's Lender of last resort Regime for the Twenty-First Century.

Kumbhakar, S., \& Lovell, K. (2000). Stochastic Frontier Analysis. Cambridge University Press, Cambridge, UK.

Malmquist S. (1953). Index numbers and indifference surfaces. Trabajos de Estadística, 4, 209-242.

Mendoza, C. (2009). Estudio sobre Protección para los Usuarios del Sistema Financiero. Central American Business Intelligence, 26. 
Pasiouras, F. (2008). International evidence on the impact of regulations and supervision on banks' technical efficiency: An application of two-stage data envelopment analysis. Review of Quantitative Finance and Accounting, 30(2), 187-223. https://doi.org/10.1007/s11156-007-0046-7

Pasiouras, F., Tanna, S., \& Zopounidis, C. (2009). The impact of banking regulations on banks' cost and profit efficiency: Cross-country evidence. International Review of Financial Analysis, 18(5), 294-302. https:// doi.org/10.1016/j.irfa.2009.07.003

Peltzman, S. (1976). Toward a More General Theory of Regulation. Journal of Law and Economics, 19(2), 211-240.

Pirateque, J., Piñeros, J., \& Mondragón (2013). Eficiencia de los establecimientos Bancarios (EB): una aproximación mediante modelos DEA. Borradores de Economía $\mathrm{N}^{\circ} 798$, 1-39.

Portela, M., \& Thanassoulis, E. (2007). Comparative efficiency analysis of Portuguese bank branches. European Journal of Operational Research, 177(2), 1275-1288. https://doi.org/10.1016/j.ejor.2006.01.007

Posner, R. A. (1974). Theories of Economic Regulation. The Bell Journal of Economics and Management Science, 5(2), 335-358.

Rodríguez, W., \& Rodríguez, O. (2014). La eficiencia técnica de los bancos durante la crisis Caso aplicado a la rentabilidad de la banca comercial en México y Chile. Contaduría y Administración, 59(1), 95-122. https:// doi.org/10.1016/S0186-1042(14)71245-8

Sathye, M. (2001). X-efficiency in Australian banking: An empirical investigation. Journal of Banking and Finance, 25(3), 613-630. https://doi.org/10.1016/S0378-4266(00)00156-4

Server, R., \& Melián, A. (1999). Estrategias competitivas y eficiencia de las secciones de crédito en la Comunidad Valenciana. Ciriec-España, 32, 39-83.

Stigler, G. J. (1971). The Theory of Economic Regulation. Bell Journal of Economics, 2(1), 3-21.

Torres, Z., Navarro, J., \& Gómez, R. (2010). El sector bancario en México: un análisis a través de la envolvente de datos. Investigación Administrativa, 106(39), 97-113. http://www.sepi.escasto.ipn.mx/Revista/Documents/revistas/ RIA106.pdf

Zaim, O. (1995). The effect of financial liberalization on the efficiency of Turkish commercial banks. Applied Financial Economics, 5(4), 257-264.

\section{Notas}

* Artículo de investigación científica.

Licencia Creative Commons CC BY 4.0 